While the case is of undoubted interest, it concerns me that the "Conclusions" section of the structured abstract states that: "SPECT is effective in the diagnosis of neuropsychiatric disorders such as $O C D$, and the pathological changes in brain metabolism detected by SPECT may be reversed by both drug therapy and psychotherapy". Clearly these conclusions, irrespective of their validity, cannot be supported by the data presented. Firstly, the description of SPECT changes in a single case of OCD hardly justifies the assertion that SPECT is "effective in the diagnosis"; OCD remains a clinical diagnosis, and whether pathognomonic blood flow changes will ever be demonstrable is highly questionable. Secondly, while it is true that the patient described improved clinically with treatment, it is unclear from a single case study whether this was because of the treatment offered (and if so, what elements were effective), or despite it. Thirdly, the authors do not report a repeat post-recovery SPECT to justify the conclusion that "changes in brain metabolism" were indeed reversed.

All this might appear pedantic, but it is imperative that conclusions are justified in terms of data presented. Given that many readers will confine themselves to a perusal of the abstract of many articles, it is doubly important that the conclusions in the abstract are accurate. The introduction of structured abstracts in the $B J P$ is a welcome development, but the quality of the abstract is by no means ensured through having structure. I suggest reviewers been asked specifically to assess the coherence and validity of the structured abstracts in submitted papers.

SnMPsON, S. \& BALDWIN, B. (1995) Neuropsychiatry and SPECT of an acute obsessive-compulsive syndrome patient. British Journal of Psychiatry, 166, 390-392.

The University of Western Australia

D.J. Castle

35 Mills Stree

Bentley, WA 6102

\section{Psychosocial outcome of liver transplant}

SR: We read Collis et al's (1995) study with interest as we also published a study describing the psychosocial outcome of liver transplantation (Howard et al, 1994). We undertook a study of similar cross-sectional design but some of our results differed appreciably from those of Collis et al.

Our study focused on the outcome of transplant recipients transplanted for end-stage alcoholic cirrhosis $(n=20)$. We interviewed subjects in detail extensively, but also administered several standardised questionnaires to these patients and other liver transplant patient controls $(n=54)$. Our study found levels of psychiatric morbidity in both groups similar to those found by Collis et al and we agree with their conclusion that the prevalence of psychiatric disorder in post-transplantation patients is comparable with that of general medical patients.

Although Collis et al speculated that alcoholic patients may have a particularly high risk of psychiatric morbidity on the basis of their finding that three of four alcoholic patients were CIS (Clinical Interview Schedule) cases, we found no evidence of this. There was no significant difference between the alcoholic patients and transplant controls on any measure of psychosocial outcome except for a poorer perception of health status by the alcoholics, and only one alcoholic patient of 18 administered the CIS obtained a score compatible with psychiatric caseness. There was also no significant difference between the two groups on all measures of physical outcome. Only one other group has reported on the psychological outcome of alcoholic transplant patients (Beresford et al, 1992) and they also found no difference in the prevalence of depressed mood between alcoholic patients and transplant controls (although no standardised measures were used in this study).

It is of interest that most alcoholic patients returned to regular alcohol drinking after a period of abstinence (usually of several months), but at lower levels than before the transplant.

Possible explanations for the different findings of Collis's study and ours include different selection criteria for transplantation, and differences in social support and demographic characteristics (such as social class), but we suspect that the longer period of follow-up in our study (our patients were assessed 1 to 6 years after transplantation) is most relevant. Quality of life is particularly impaired in the first year after transplantation compared with subsequent years (Lowe et al, 1990).

We conclude that the evidence to date suggests that alcoholic liver transplant recipients do not have higher levels of psychiatric morbidity than other liver transplant patients but they remain vulnerable to alcohol abuse in the medium to long term. The need for specialist input from psychiatric and alcohol services should therefore be anticipated by transplant units and liaison psychiatrists if patients with alcohol problems continue to be transplanted in increasing numbers. 
Beresford T.P., Schwartz, J., Wilson., D., et al (1992) The short term psychological health of alcoholic and nonalcoholic liver transplant recipients. Alcoholism: Clinical and Experimental Research, 16, 996-1000.

Colus, I., Burroughs, A., Rolles, K., et al (1995) Psychiatric and social outcome of liver transplantation. British Journal of Psychiatry, 166, 521-524.

Howard, L., FAHY, T., Wong, P., et al (1994) Psychiatric outcome in alcoholic liver transplant patients. Quarterly Journal of Medicine, 87, 731-736.

LOWE, D., O'Grady, J., McEwan, J., et al (1990) Quality of life following liver transplantation: a preliminary report. Journal of the Royal College of Physicians, 24, 43-46.

L.M. HOWARD

T. FAHY

The Maudsley Hospital

London SE5 8AZ

\section{Costs of community psychiatric nurse teams}

SIR: McCrone et al's paper (August 1994) analyses data from the Greenwich service reported in the preceding paper in the same issue by Muijen et al and concludes that "... the CST [community support team] is a cost-effective alternative to generic CPN [community psychiatric nurse team] arrangements". Over the 18 months studied the generic group is claimed to cost an average of $£ 110$ more per patient per week.

Close examination of the data does not appear to support this conclusion. This small study demonstrated remarkably few differences in either clinical and social outcome or in reduction in hospital care despite markedly increased CPN contact in the intervention group. Where, then, do the cost savings arise?

The major cost advantage to the CST group is accounted for by lower accommodation costs $£ 148$ per patient per week as opposed to $£ 269$ for the generic group. This is presumably due to the higher number of generic patients who were living in specialist care settings ( $22 \%$ c.f. $2 \%$ at intake and $22 \%$ c.f. $3 \%$ at follow up according to Muijen et al). The figures are harder to disentangle in the McCrone et al paper but they state - "More clients from the generic group lived in specialist care settings (homes, hostels or hospital) both at referral $(15 \%)$ and 18 months later (23\%)." Direct treatment costs, on the other hand, are marginally greater in the CST group (i.e. subtracting accommodation costs from total costs) - $£ 137$ per patient per week c.f. $£ 126$ in generic care.

There is no reason to assume from these two papers that the differences in accommodation costs are anything other than an artefact of the randomisation. The failure to acknowledge this and make adequate allowances for it in the paper's discussion obscures the study findings. McCrone et al have conducted their economic analysis following Beecham \& Knapp's (1990) four principles of cost evaluation (which emphasise the need for comprehensives). These four principles are probably essential for costing across widely differing procedures and disorders (e.g. comparing the cost benefits of hip replacements against diabetic out-patient clinics). Their application in RCTs of a defined patient population, however, obscures more than it illuminates.

Judgement needs to be exercised in the conduct of economic evaluations in mental health studies if they are not to lead to serious misunderstandings as I believe they have in this paper.

BeECHAM, J. \& KNAPP, M. (1990) Costing mental health services. Psychological Medicine, 20, 893-908.

McCrone. P., BeECham, J. \& KNAPP, M. (1994) Community psychiatric nurse teams: cost-effectiveness of intensive support versus generic care. British Journal of Psychiatry. 165, 218-221. Muden, M., CoOney, M., Strathdee, G., et al (1994) Community psychiatric nurse teams: intensive support versus generic care. British Journal of Psychiatry, 165, 211-217.

\section{St Georges Hospital Medical School London SW17 ORE}

AUTHOR's REPLY: Burns has confused the shortterm (0-6 months) and longer-term (0-18 months) findings from our cost-effectiveness study of the Greenwich service.

The quote from our paper in his first paragraph is taken out of context. The words which precede the clause he quotes are "In the short term, therefore ...". And the sentence which follows the quote is: "Beyond the short term, the CST did not have a cost or cost-effectiveness advantage". We categorically did not say that the CST was more cost-effective than generic CPN services over the 18-month period.

What happened in the short term (0-6 months) to give the significant cost advantage to the CST? Accommodation and hospital costs were significantly lower. When account is taken of the fact that the CST group looked as if it made use of less specialised accommodation in the pre-referral period, we still find the CST to have a cost advantage in the first six months of the intervention. ('Net costs' between pre-referral and 0-6 months showed CST costs were still lower than generic costs; $P<0.05)$. Over the whole research period (0-18 months), accommodation costs were lower for the CST group $(P<0.001)$, but other costs counter-balanced this advantage to give the 